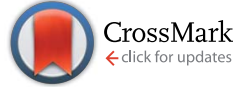

Cite this: Anal. Methods, 2014, 6, 6232

Received 11th March 2014 Accepted 29th April 2014

DOI: $10.1039 / c 4 a y 00614 c$

\section{Automated enzyme-linked immunosorbent assay using beads in a single tip (BIST) technology coupled with a novel anchor protein for oriented antibody immobilization}

\author{
Takeshi Ikeda, ${ }^{\text {*a }}$ Tetsuya Ueda, ${ }^{\mathrm{b}}$ Hideji Tajima, ${ }^{\mathrm{b}}$ Kiyoshi Sekiguchic ${ }^{\mathrm{a}}$ and Akio Kuroda ${ }^{\mathrm{ac}}$
}

www.rsc.org/methods

An automated enzyme-linked immunosorbent assay (ELISA) system was developed using beads in a single tip (BIST) technology. Sandwich ELISA was performed on glass beads functionalized with a capture antibody via the fusion of antibody-binding streptococcal protein $G$ and a silica/glass-binding protein Si-tag. Our system enables rapid, sensitive detection of antigens.

The enzyme-linked immunosorbent assay (ELISA) is a popular analytical format for detecting and quantifying target substances. In a sandwich ELISA, a capture antibody is generally immobilized by physical adsorption onto the surface of 96- or 384-well microplates. However, when antibodies are immobilized on a solid surface, their binding activity usually decreases. ${ }^{1}$ This reduced binding activity is ascribed to the random orientation and steric hindrance associated with immobilization of the antibody molecules on the solid phase. ${ }^{1}$ Therefore, a method for oriented immobilization of functional antibodies that will increase immunoassay sensitivity is needed.

We recently developed a method for immobilization of proteins on silica $\left(\mathrm{SiO}_{2}\right)$ surfaces using a silica-binding affinity tag, Si-tag. ${ }^{2,3}$ Silica is the most widely used solid support because of its mechanical and chemical stability, ease of synthesis, and cost effectiveness. The Si-tag is a 273-amino acid fusion tag that binds strongly to unmodified silica surfaces (e.g., silica particles, glass slides, and silicon wafers with an insulating oxide layer). ${ }^{2,4-6} \mathrm{Si}$-tagged fusion proteins can be bound to a silica surface by simply mixing them with or spotting them onto a target, even if there are high concentrations of salt,

\footnotetext{
${ }^{a}$ Department of Molecular Biotechnology, Graduate School of Advanced Sciences of Matter, Hiroshima University, 1-3-1 Kagamiyama, Higashi-Hiroshima, Hiroshima 739-8530, Japan. E-mail: ikedatakeshi@hiroshima-u.ac.jp; Fax: +81 82424 7047; Tel: +81824247047

${ }^{b}$ Precision System Science Co., Ltd., 88 Kamihongo, Matsudo, Chiba 271-0064, Japan 'Siliconbio Inc., 3-10-31 Kagamiyama, Higashi-Hiroshima, Hiroshima 739-0046, Japan
}

detergent, and non-ionic chaotrope (e.g., $8 \mathrm{M}$ urea) present in the protein solution. ${ }^{7,8}$

In order to achieve oriented antibody immobilization on a silica surface, in this study we constructed a fusion protein consisting of a Si-tag and immunoglobulin-binding streptococcal protein G (SpG), namely Si-tagged SpG. SpG is a cell wall component of groups $\mathrm{C}$ and $\mathrm{G}$ streptococcal bacteria that can bind many immunoglobulin $\mathrm{G}$ (IgG) molecules. ${ }^{\mathbf{9}, 10}$ SpG interacts with IgG predominantly via the Fc region ${ }^{\mathbf{1 0 , 1 1}}$ and therefore does not interfere with the binding of the antigen, which occurs on the distal ends of the Fab regions. By using Si-tagged SpG as an anchor protein, antibodies can be immobilized on a silica/glass surface in an oriented manner.

In this proof-of-principle study, we adopted glass beads as the solid phase for antibody immobilization and developed an automated ELISA system using the recently reported beads in a single tip (BIST) technology. ${ }^{12}$ BIST technology utilizes a capillary-shaped tip containing up to $481 \mathrm{~mm}$ diameter beads in an array (Fig. 1). The bead-containing tip is vertically connected to an automated pipetting robot (Purelumn system; Precision System Science Co., Ltd., Chiba, Japan) that performs programmable, sequential cycles of aspirating and dispensing solutions into and out of the tip. Therefore, desired assays can be performed automatically when appropriate receptors for an analyte of interest are immobilized on the beads in the tip. Here, we report a BIST-based automated ELISA system (designated BIST-ELISA) coupled with Si-tagged SpG for oriented antibody immobilization on glass beads (Fig. 1).

To construct the expression plasmid for Si-tagged SpG, the DNA fragment encoding the B1 domain (55 amino acids) of $\mathrm{SpG}^{\mathbf{1 3}}$ was synthesized with a $5^{\prime}$-TA overhang at the upstream end and a $5^{\prime}$-GATC overhang at the downstream end. The $5^{\prime}$-TA and 5'-GATC overhangs are identical to the cohesive ends of NdeI- and BamHI-digested fragments, respectively. The synthesized fragment was ligated with NdeI-BamHI-digested pET-SpA-Sitag $^{\mathbf{1 4}}$ to construct pET-SpG-Sitag, which carries the gene encoding the $38.2 \mathrm{kDa}$ recombinant protein consisting of the IgG-binding B1 domain of SpG and a C-terminal Si-tag. 


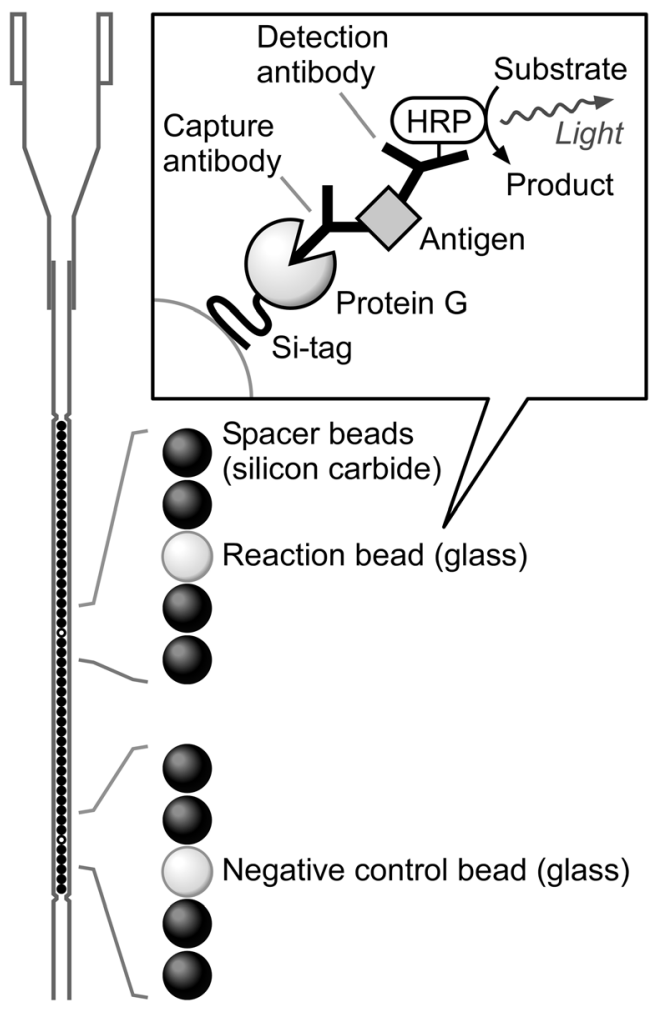

Fig. 1 Schematic representation of BIST technology and the BISTELISA. Beads ( $1 \mathrm{~mm}$ diameter) are arrayed in a capillary-shaped tip. Typically, one reaction bead is contained in a single tip with one negative control bead and 46 spacer beads. The upper part of the tip is designed to be connected to the automated pipetting robot as well as a standard $1 \mathrm{~mL}$ micropipette. Sandwich ELISA is performed on the reaction bead with IgG immobilized via Si-tagged SpG as the capture antibody. The Si-tag is represented as a curved line in the inset box because it is not a canonical globular protein. ${ }^{3}$

Escherichia coli Rosetta(DE3)pLysS (Novagen/Merck KGaA, Darmstadt, Germany) was transformed with pET-SpG-Sitag. The transformants were cultivated at $37{ }^{\circ} \mathrm{C}$ in $2 \times \mathrm{YT}_{\text {medium }}{ }^{15}$ supplemented with $50 \mu \mathrm{g} \mathrm{mL}^{-1}$ of carbenicillin and $30 \mu \mathrm{g} \mathrm{mL}^{-1}$ of chloramphenicol. When the culture reached an optical density at $600 \mathrm{~nm}$ of $0.5,1 \mathrm{mM}$ isopropyl- $\beta$-D-thiogalactopyranoside was added to the medium. After an additional $8 \mathrm{~h}$ of cultivation at $28{ }^{\circ} \mathrm{C}$, cells were harvested by centrifugation and disrupted by sonication. The recombinant protein was purified by cation exchange chromatography on a Poros HS/20 column (Applied Biosystems/Thermo Fisher Scientific Inc., Waltham, MA, USA) with a linear gradient from 0 to $1 \mathrm{M} \mathrm{NaCl}$ in $20 \mathrm{mM}$ Tris-HCl buffer ( $\mathrm{pH}$ 8.0). Fractions containing the recombinant protein were divided into small aliquots and stored at $-80{ }^{\circ} \mathrm{C}$ until use. Because the Si-tag binds to glass surfaces, ${ }^{2}$ all protein manipulations were carried out using polypropylene tubes and tips.

The recombinant Si-tagged SpG was expressed in soluble form in $E$. coli at $28{ }^{\circ} \mathrm{C}$. Because the Si-tag is a highly basic protein with a theoretical isoelectric point of $10.9,{ }^{2}$ cation exchange chromatography efficiently purified the Si-tagged SpG to apparent homogeneity (data not shown). To test the functionality of the recombinant protein, we performed glass and IgG-binding assays as follows (unless otherwise specified, all manipulations were performed at room temperature). Soda glass beads $\left(1.0 \mathrm{~mm}\right.$ diameter with a surface roughness $\left[R_{\mathrm{a}}\right]$ value of $\sim 90 \mathrm{~nm}$; prepared by Tsubaki Nakashima Co., Ltd., Nara, Japan) were incubated for $30 \mathrm{~min}$ with gentle horizontal rotation in a $200 \mu \mathrm{L}$ aliquot of $0.1 \mathrm{mg} \mathrm{mL} \mathrm{mL}^{-1}$ Si-tagged SpG solution in $25 \mathrm{mM}$ Tris-HCl buffer ( $\mathrm{pH} 9.0$ ) containing $0.5 \mathrm{M}$ $\mathrm{NaCl}$ and $0.5 \%(\mathrm{v} / \mathrm{v})$ Tween 20 . After washing three times with $200 \mu \mathrm{L}$ of phosphate-buffered saline (PBS), the beads were reacted for $30 \mathrm{~min}$ with $100 \mu \mathrm{L}$ of $1: 50000$ horseradish peroxidase (HRP)-conjugated mouse $\mathrm{IgG}_{1}$, which was prepared by labeling $200 \mu \mathrm{g}$ of mouse $\operatorname{IgG}_{1}$ with HRP using a Peroxidase Labeling Kit-SH (Dojindo Laboratories, Kumamoto, Japan). When incubated with a chemiluminescent substrate solution for HRP (SuperSignal West Femto Maximum Sensitivity Substrate; Thermo Fisher Scientific Inc.), the beads showed much higher chemiluminescence intensity than those prepared without Si-tagged SpG $\left(2.5[ \pm 0.3] \times 10^{5}\right.$ vs. $1.3[ \pm 0.1] \times 10^{3}$ relative luminescence units [RLU], mean \pm standard deviation of triplicate measurements). Without HRP-conjugated mouse $\mathrm{IgG}_{1}$, the beads showed less than $200 \mathrm{RLU}$. These results clearly indicate that Si-tagged SpG binds to both silica/glass surfaces and IgG and functions as an anchor protein suitable for antibody immobilization.

Next, we performed BIST-ELISA using glass beads functionalized with an antibody via Si-tagged SpG. In this proof-ofprinciple study, human thyroid-stimulating hormone (TSH; 8.3 IU mg ${ }^{-1}$; Scipac Ltd., Sittingbourne, UK) was used as a model antigen. The serum concentration of $\mathrm{TSH}$ is an important indicator used for early detection of hyper- and hypothyroidism. Anti-TSH monoclonal mouse IgG $_{1}$ (10-T25C; Fitzgerald Industries International, Acton, MA, USA) was used as the capture antibody. Another anti-TSH mouse IgG $_{1}$ (10-T25B; Fitzgerald Industries International) was conjugated with HRP as described above and used as the detection antibody. Up to 20 glass beads were incubated in the Si-tagged SpG solution as described above. After washing three times with $200 \mu \mathrm{L}$ of PBS, the beads were incubated with gentle horizontal rotation overnight at $4{ }^{\circ} \mathrm{C}$ in a $200 \mu \mathrm{L}$ aliquot of $10 \mu \mathrm{g} \mathrm{mL}{ }^{-1}$ solution of capture antibody in $50 \mathrm{mM}$ citrate buffer ( $\mathrm{pH} 5.0$ ) containing $0.15 \mathrm{M} \mathrm{NaCl}$ (pH 5 is the optimal $\mathrm{pH}$ for the binding of IgG to SpG). ${ }^{16}$ Glass beads functionalized with the antibody via Si-tagged SpG were then blocked with $1 \%(\mathrm{w} / \mathrm{v})$ bovine serum albumin in PBS for $2 \mathrm{~h}$. One of the functionalized beads (designated the reaction bead) was placed into a BIST tip with one negative control bead and 46 spacer beads (glass and silicon carbide beads $[1.0 \mathrm{~mm}$ diameter; prepared by Tsubaki Nakashima Co., Ltd.] blocked with $1 \%$ $[\mathrm{w} / \mathrm{v}]$ bovine serum albumin in PBS) in the arrangement shown in Fig. 1. The tip, containing a total of 48 beads, was then connected to the automated pipetting robot, which can handle up to 6 tips simultaneously. A 10-well cartridge containing solutions for the assay was also placed in the designated area inside the robot. The subsequent procedures were performed automatically by the robot. First, the beads within the tip were washed by 10 cycles of automatic aspiration and dispensing of $100 \mu \mathrm{L}$ of wash buffer (PBS containing $0.05 \%$ [v/v] Tween 20 and 
0.05\% [v/v] Adeka Nol LG-299 antifoaming agent [Adeka Corporation, Tokyo, Japan]). Second, the beads were reacted with a mixture of $50 \mu \mathrm{L}$ of TSH solution of various concentrations and $50 \mu \mathrm{L}$ of $1: 25000 \mathrm{HRP}$-conjugated detection antibody by 300 cycles of aspiration and dispensing of the mixture. Third, the beads were washed with wash buffer as described above. The washing step was repeated two more times with $100 \mu \mathrm{L}$ aliquots of wash buffer. Finally, the tip was automatically detached from the pipetting robot. Our system allows for completion of overall reactions (a total of 340 cycles of aspiration and dispensing) within $40 \mathrm{~min}$. The chemiluminescent substrate solution for HRP was manually aspirated into the tip with a $1 \mathrm{~mL}$ micropipette, and the lower opening of the tip was sealed with putty (XX-VCS35; Terumo Corporation, Tokyo, Japan). The chemiluminescence of each bead was then measured using a photodetection unit for BIST (BISTnner; Precision System Science Co., Ltd.). ${ }^{12}$

The reaction beads showed a linear increase in chemiluminescence that was proportional to the TSH concentration (Fig. 2). These results demonstrate that the BIST system coupled with the antibody immobilized via Si-tagged SpG is applicable to automated ELISA. The glass beads functionalized with the antibody via Si-tagged SpG showed about 3-fold higher chemiluminescence than those with the physically adsorbed antibody, which was prepared by incubating glass beads with $10 \mu \mathrm{g}$ $\mathrm{mL}^{-1}$ of capture antibody in PBS overnight at $4{ }^{\circ} \mathrm{C}$ (Fig. 2). The higher intensity obtained by our method can be attributed to the well-ordered orientation of the immobilized antibody molecules, which enhances the steric accessibility of the antigen-binding sites to the soluble antigen, as demonstrated in our previous work. ${ }^{14}$ Our TSH assay was highly sensitive, with detection and quantification limits of 0.04 and $0.08 \mu \mathrm{IU} \mathrm{mL}{ }^{-1}$,

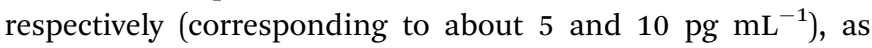
determined from the standard deviation of the chemiluminescence intensity of the blank. ${ }^{17}$ These values are much

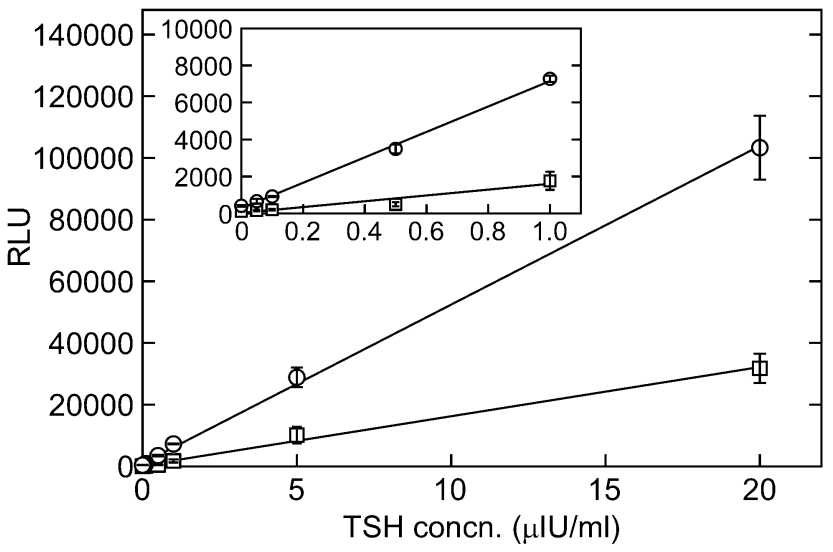

Fig. 2 TSH detection by BIST-ELISA. Sandwich ELISA was performed on a glass bead in the tip using mouse $\lg _{1}$ immobilized on the bead via Si-tagged SpG (circles) or via physical adsorption (squares) as the capture antibody. The inset shows the plot at low TSH concentrations. Values are means \pm standard deviations of raw RLU values from triplicate measurements. lower than the normal reference range for TSH $(0.3-3.0 \mu \mathrm{IU}$ $\mathrm{mL}^{-1}$ according to the guidelines of the American Association of Clinical Endocrinologists; ${ }^{18}$ reference ranges may vary slightly between laboratories). In contrast, when glass beads with the physically adsorbed antibody were used, the detection and quantification limits for TSH $\left(0.2\right.$ and $0.3 \mu \mathrm{IU} \mathrm{mL}^{-1}$, respectively) were 4-5 times higher than those obtained with $\mathrm{Si}$ tagged SpG, confirming the advantage of this anchor protein for antibody immobilization.

Additional benefits of the BIST-ELISA system include the automation of tedious liquid handling steps associated with conventional ELISA as well as reduction of assay time. Furthermore, the automated assay provides better precision with less operator dependence. To further improve our system, full automation of the assay (including chemiluminescence detection) is in progress. In addition, multiplex analyte detection is also being developed by placing several reaction beads in a single tip.

In conclusion, we developed a versatile and sensitive automated ELISA system by coupling BIST with an antibody via Sitagged SpG. Our BIST-ELISA system allows for the automated detection and quantification of any analyte by simply incorporating appropriate antibodies.

\section{Acknowledgements}

This work was supported in part by the Industrial Technology Research Grant Program in 2009 from the New Energy and Industrial Technology Development Organization (NEDO) of Japan (no. 09C46130a to T.I.).

\section{References}

1 B. Lu, M. R. Smyth and R. O'Kennedy, Analyst, 1996, 121, 29R-32R.

2 K. Taniguchi, K. Nomura, Y. Hata, T. Nishimura, Y. Asami and A. Kuroda, Biotechnol. Bioeng., 2007, 96, 1023-1029.

3 T. Ikeda and A. Kuroda, Colloids Surf., B, 2011, 86, 359-363.

4 M. Fukuyama, S. Yamatogi, H. Ding, M. Nishida, C. Kawamoto, Y. Amemiya, T. Ikeda, T. Noda, S. Kawamoto, K. Ono, A. Kuroda and S. Yokoyama, Jpn. J. Appl. Phys., 2010, 49, 04DL09.

5 M. Fukuyama, M. Nishida, Y. Abe, Y. Amemiya, T. Ikeda, A. Kuroda and S. Yokoyama, Jpn. J. Appl. Phys., 2011, 50, 04 DL07.

6 S. Yamatogi, Y. Amemiya, T. Ikeda, A. Kuroda and S. Yokoyama, Jpn. J. Appl. Phys., 2009, 48, $04 \mathrm{C} 188$.

7 T. Ikeda, K. Ninomiya, R. Hirota and A. Kuroda, Protein Expression Purif., 2010, 71, 91-95.

8 T. Ikeda, K. Motomura, Y. Agou, T. Ishida, R. Hirota and A. Kuroda, Protein Expression Purif., 2011, 77, 173-177.

9 B. Åkerström, T. Brodin, K. Reis and L. Björck, J. Immunol., 1985, 135, 2589-2592.

10 L. Björck and G. Kronvall, J. Immunol., 1984, 133, 969-974.

11 M. Tashiro and G. T. Montelione, Curr. Opin. Struct. Biol., 1995, 5, 471-481. 
12 Y. Kagawa, M. Hiraoka, Y. Miyashita-Hatano, M. ShishidoOki, M. Yoshida, S. Kondou, M. Sugiura, K. SawakamiKobayashi, M. Takahashi, H. Tajima and M. Yohda, J. Biosci. Bioeng., 2010, 110, 505-508.

13 S. R. Fahnestock, P. Alexander, J. Nagle and D. Filpula, J. Bacteriol., 1986, 167, 870-880.

14 T. Ikeda, Y. Hata, K. Ninomiya, Y. Ikura, K. Takeguchi, S. Aoyagi, R. Hirota and A. Kuroda, Anal. Biochem., 2009, 385, 132-137.
15 M. R. Green and J. Sambrook, Molecular Cloning: A Laboratory Manual, Cold Spring Harbor Laboratory Press, New York, 4th edn., 2012.

16 B. Åkerström and L. Björck, J. Biol. Chem., 1986, 261, 1024010247.

17 L. A. Currie, Pure Appl. Chem., 1995, 67, 1699-1723.

18 H. J. Baskin, R. H. Cobin, D. S. Duick, H. Gharib, R. B. Guttler, M. M. Kaplan and R. L. Segal, Endocr. Pract., 2002, 8, 457-469. 